\title{
Cellular Communication Propagation at Drone around Building Environment with Single Knife Edge at $10 \mathrm{GHz}$
}

\author{
Andrita Ceriana Eska \\ Electrical Engineering Department, University of Jember \\ Jl. Kalimantan 37 Kampus Tegalboto, POS 159 Jember, Indonesia \\ Corresponding email: andritacerianaeska@gmail.com
}

Received 14 August 2020, Revised 20 January 2021, Accepted 8 February 2021

\begin{abstract}
The drone communication systems used a cellular network for controlling a drone from a long distance. That communication propagations between drone and base station were analyzed. The drone moved at the track around building environment. That environment used variations in building height. The communication propagation around building environment caused diffraction mechanism. Single knife edge method is used for that diffraction mechanism. The frequency of communication used $10 \mathrm{GHz}$. That frequency was influenced by atmospheric attenuation. This research was using some variations such as height of drone track location, transmitter power, and AMC (Adaptive Modulation Coding). MCS (Modulation Coding Scheme) was used AMC such as QPSK, 16 QAM, and 64 QAM. Some result was obtained at this research consist of LOS and NLOS distance, SNR, MCS probability, and percentage of drone coverage. NLOS propagation was caused by building height. The SNR value become increase when higher at drone position, such as drone was moving at 20 meters with height of flying drone $80 \mathrm{~m}$ and transmitter power $30 \mathrm{dBm}$ obtained SNR $38.21 \mathrm{dBm}$. That SNR is affected AMC, so a higher SNR value increases AMC. The drone's coverage $100 \%$, with a height of flying drone 80 meters and transmitter power of $30 \mathrm{dBm}$. That condition showed more increasing coverage percentage than $64.8 \%$ for height of flying drone 20 meters and transmitter power $30 \mathrm{dBm}$. That result showed that more drone height increased of coverage percentage, probability modulation, and SNR value.
\end{abstract}

Keywords - AMC, $10 \mathrm{GHz}$, drone, building

Copyright $\odot 2021$ JURNAL INFOTEL

All rights reserved.

\section{INTRODUCTION}

The drone was continually evolving, such as security, company, military, etc. This research is modeled with simulation for drone communication. That drone communication used a cellular network. Base Station (BS) location is placed everywhere. The cellular network was constantly evolving for communication. Some cellular communication researches related with super high frequency or too high frequency such as propagation for mobile communication around tree used OFDM-QAM at 10 $\mathrm{GHz}$ [1], propagation from outdoor to indoor with AMC using $10 \mathrm{GHz}$ [2], and outdoor to indoor condition for path loss at picocell and femtocell [3]. Some researches related to millimeter-wave at femtocell, such as resource allocation schemes for cognitive LTE-A femtocells [4]. Femtocell enhanced multitarget spectrum allocation strategy in LTE-A Het
Nets [5], RBS femtocell propagation at street pole lamp used $10 \mathrm{GHz}$ frequency [6]. communication systems influenced code rate at RBS femtocell at street pole lamp [7].

Some researches were related with millimeter wave communication propagation such as propagation measurement at indoor used millimeterwave for wireless network 5G [8], determination location for mobile station around the building with AoA method at $47 \mathrm{GHz}$ frequency [9], measurement at base station diversity for $5 \mathrm{G}$ using millimeter-wave with $73 \mathrm{GHz}$ [10], the millimeter-wave network for self-backhauling relay nodes and centralized transmission coordination [11], millimeter-wave for 5G communication at small area [12], millimeterwave communication of $5 \mathrm{G}$ at wireless network [13], propagation was depended with angle for cellular and wireless communication [14], self-backhauling with 
flexible reuse of the resources for access and backhaul in street scenario with $5 \mathrm{G}$ network [15], multipath effect around the building environment for mobile communication was used $47 \mathrm{GHz}$ frequency [16], and performance of in-band self-backhauling with integrated access and backhaul in a real-life street canyon scenario for $5 \mathrm{G}$ systems [17].

The researches of cellular communication about cellular frequency were constantly evolving more increase. This research was based on drone control from long distances. That communication propagations were analyzed between drone and base station. The communication between drone and cellular network is used for data transmission such as GPS, video, picture, sensors, etc. Base Station (BS) used some RBS (Radio Base Stations). SNR value can be obtained by drone communication with cellular network propagation, and the value affected AMC (Adaptive Modulation Coding) mechanism. This research used AMC, such as AMC, for mobile station communication at the train [18].

Some diffraction mechanisms are used such as the single knife-edge method, Epstein-Peterson diffraction method, Japanese method, Deygout method, Giovanelli Knife Edge, etc. That diffraction of propagation communication caused the SNR value more decrease. Some researches were related to that diffraction, such as mobile communication systems with diffraction propagation around the building environment [19] and mobile communication systems around the tree that used the Giovanelli Knife Edge method with $2.3 \mathrm{GHz}$ frequency [20]. Some researches were related with OFDM at mobile communication systems such as OFDM and OFDMA with DFT based [21], edge windowing for communication systems with OFDM based [22], and algorithm allocation for OFDM systems [23].

This research modeled the communication propagation of drones with the cellular network around the building environment. The communication frequency used $10 \mathrm{GHz}$. Analysis with that frequency caused the development of spectrum frequency for cellular communication. The atmospheric attenuation affected communication. That frequency was influenced by atmospheric attenuation, such as water vapor and oxygen. The analysis of communication propagation for this research used the uplink condition. Uplink condition because of part of communication transmission. The effect of the building environment is modeled with a diffraction mechanism By using a single knife-edge method. SNR communication is obtained from LOS and NLOS propagation. NLOS condition was caused by building height. That SNR determined AMC that used MCS variation. That MCS used modulations such as QPSK, 16 QAM, and 64 QAM. Every modulation used code rate variation. This research is used parameter variation such as drone height, transmitter power, and AMC. The drone used various heights, such as 40 meters, 60 meters, and 80 meters. The transmitter power that has been used $20 \mathrm{dBm}$ and 30 $\mathrm{dBm}$. This research showed LOS and NLOS effect, SNR communication, MCS probability for AMC, and coverage percentage at the drone. This research aimed to analyze drone communication propagation around building environments with the cellular network for a remote drone from a long distance. This research was important for the development of drone communication. This research is modeled with a simulation.

\section{RESEARCH METHODS}

\section{A. Environment Model}

The drone is moved around the building environment on the straight track. The flying drone uses various heights such as 40 meters, 60 meters, and 80 meters. Figure 1 shown communication propagation of drone at 40 meters with RBS. The communication propagation of drone at 60 meters with RBS is shown in Fig. 2. While Fig. 3 showed communication propagation of drone at 80 meters with RBS. That building environment is modeled with a high variation of building that shows NLOS propagation caused by their diffraction. Single knife edge method used for calculation of diffraction mechanism [24]. Figure 4 showed single knife-edge method [25].

Figure 4 showed block diagram communication from the transmitter to receiver. Drone used various heights such as 40 meters, 60 meters, and 80 meters. The building environment and atmospheric attenuation cause the communication propagation. The receiver used RBS (Radio Base Station) with a height of 30 meters.

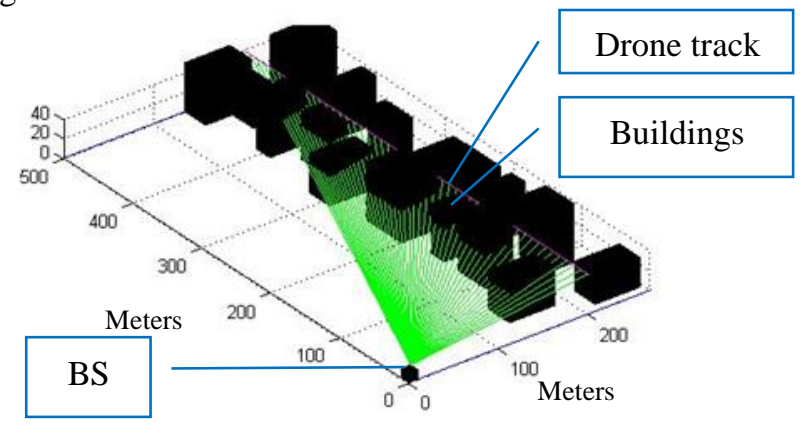

Fig. 1. Drone propagation with a height of 40 meters

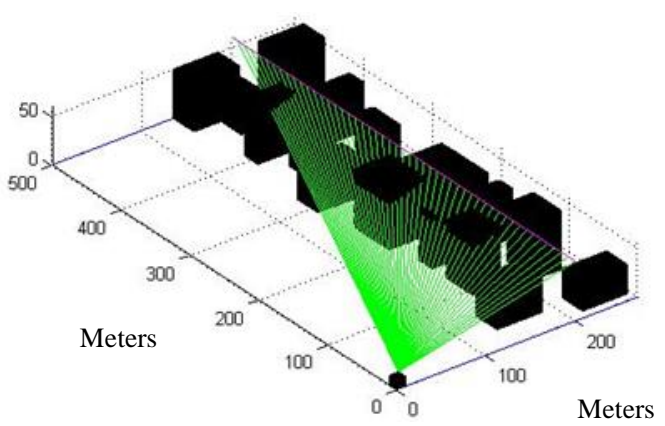


Fig. 2. Drone propagation with a height of 60 meters

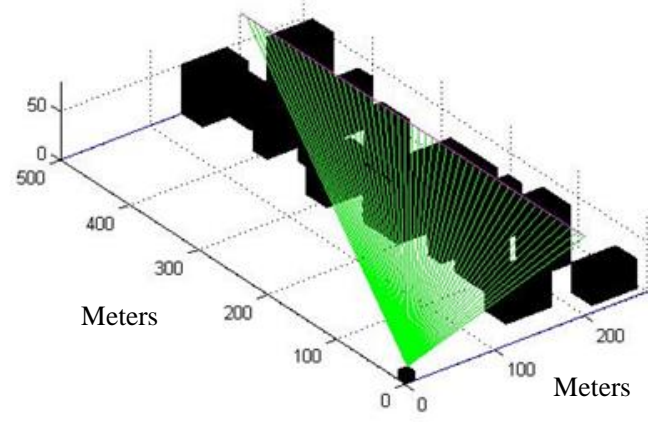

Fig. 3. Drone propagation with a height of 80 meters

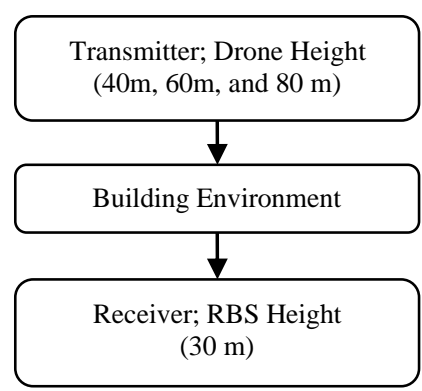

Fig. 4. Block Diagram from the transmitter to receiver

\section{B. Single Knife Edge Method}

A diffraction mechanism caused the building height. This research used a single knife-edge method for diffraction mechanism. Equation 1 showed single knife edge method. Parameter of $\lambda, h, v, d_{1}$, and $d_{2}$ was longwave (meters), high of diffraction (meters), Fresnel Kirchoff, transmitter distance through the node (meters), and receiver distance through the node (meters) [24].

$$
v=h \sqrt{\frac{d\left(d_{1}+d_{2}\right)}{\lambda d_{1} d_{2}}}=\alpha \sqrt{\frac{2 d_{1} d_{2}}{\lambda\left(d_{1}+d_{2}\right)}}
$$

Approximation to the diffraction used Fresnel Kirchoff diffraction at Fresnel Zone. Fresnel Zone is used between a transmitter and receiver. Diffraction with LOS condition, then $h$ is negative and $v$ also negative. Transmitter power variations used $20 \mathrm{dBm}$ and $30 \mathrm{dBm}$. Parameter of s, N, and SNR was signal value, noise power, and signal to noise ratio [22].

$$
S N R=\frac{S}{N}
$$

$\mathrm{N}$ parameter was showed on equation (3). Parameter of $K, B, N F, T$ was Boltzman constant, bandwidth, noise Fig., and temperature $\left(290^{\circ} \mathrm{K}\right)$ [24]. Noise Fig. used $5 \mathrm{~dB}$. Bandwidth used $5 \mathrm{MHz}$ [25].

$$
N=k T_{o} B+N F
$$

The attenuations of communication were caused by atmospheric attenuation. Atmospheric attenuations were influenced by oxygen, and water vapor could be observed int equation (4) [26]. $\gamma$ and $r_{o}$ parameters were described with gaseous attenuation and path length $(\mathrm{km})$.

AMC process based on modulation and coding scheme (MCS). MCS used modulations such as QPSK, 16 QAM, and 64 QAM [27]. The modulation of QPSK used some code rates such as 1/8, 1/5. 1/4, $1 / 3,1 / 2,2 / 3,3 / 4$, and $4 / 5$. The modulation of 16 QAM used some code rates such as $1 / 2,2 / 3,3 / 4$, and 4/5. Simultaneously, the modulation of 64 QAM used some code rates such as $2 / 3,3 / 4$, and 4/5. AMC process at communication propagation based on threshold modulation and coding scheme.

$$
A=\gamma r_{o} d B
$$

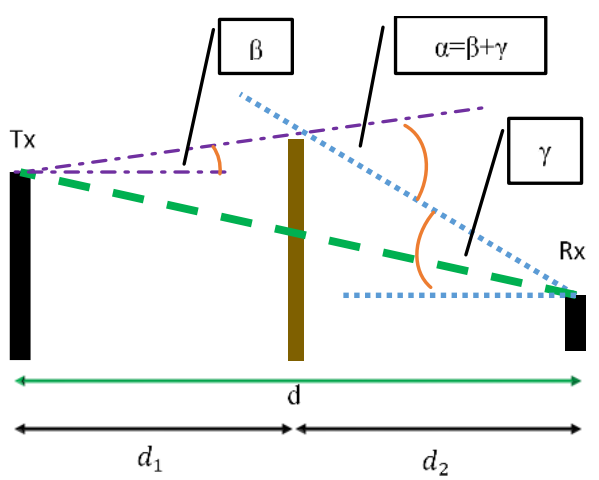

Fig. 5. Single Knife Edge Diffraction Model

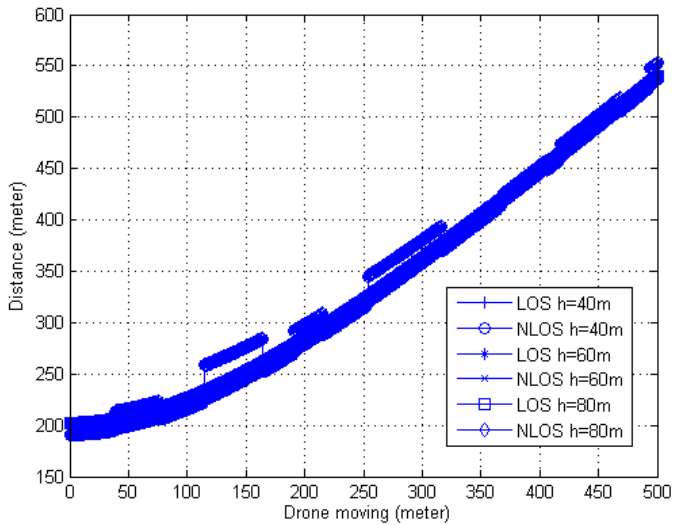

Fig. 6. The communication distance from the height of flying drones such as 40m, 60m, and 80m, shows more detail in Fig.7.

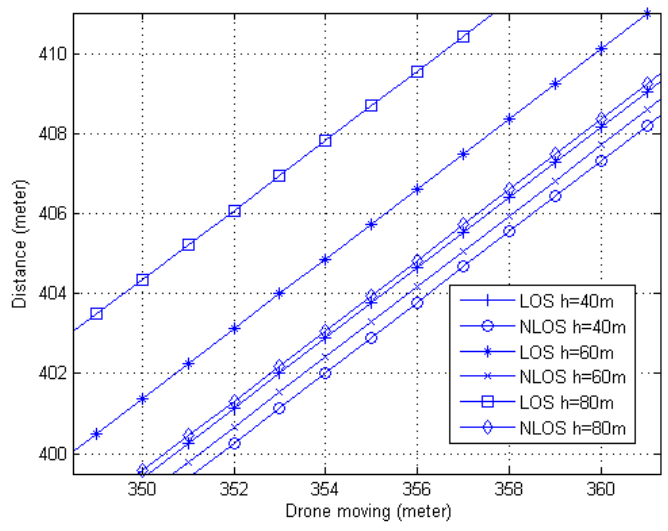

Fig. 7. Zoom in Fig. 6. 


\section{RESULTS}

This section describes the result based on the drone communication propagation. That drone communication system used a cellular network for a controlled drone from a long distance. That propagation analyzed communication between drone and base station. The communication frequency used $10 \mathrm{GHz}$. That frequency was influenced by oxygen and water vapor. The communication propagation of drone used uplink condition using various heights such as 40 meters, 60 meters, and 80 meters are used. Drone moved around the building environment on a straight track. The diffraction mechanism was caused by NLOS propagation around the building. Some analyze was used such as the height of flying drone variation, transmitter power variation, and MCS. The transmitter power used $20 \mathrm{dBm}$ and $30 \mathrm{dBm}$. That MCS used modulations such as QPSK, 16 QAM, and 64 QAM.

Figure 6 showed communication propagation of drone with LOS and NLOS condition when used transmitter power $20 \mathrm{dBm}$, for more detail showed at Fig. 7. When a drone moving at $20 \mathrm{dBm}$ is obtained, LOS distances 193.39 meters, some data, when the drone was moving at 50 meters, LOS distances obtained at 198.74 meters and NLOS distances 215.52 meters, and when the drone was moving at 400 meters, LOS distances were 433.84 meters and NLOS distances 451.52 meters.

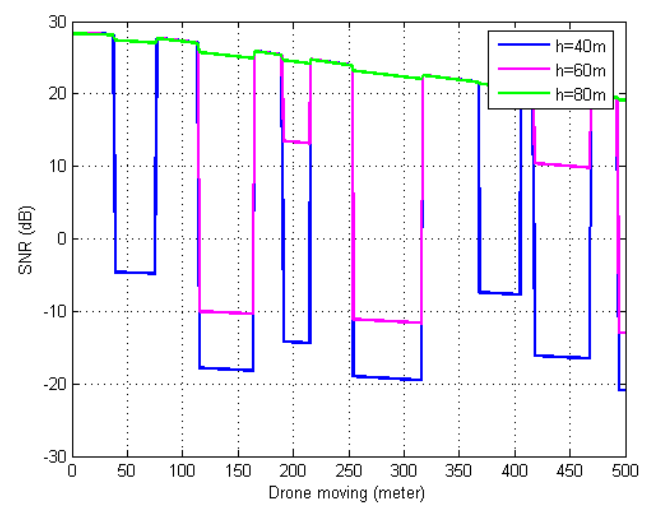

Fig. 8. The communication with transmitter power $20 \mathrm{dBm}$

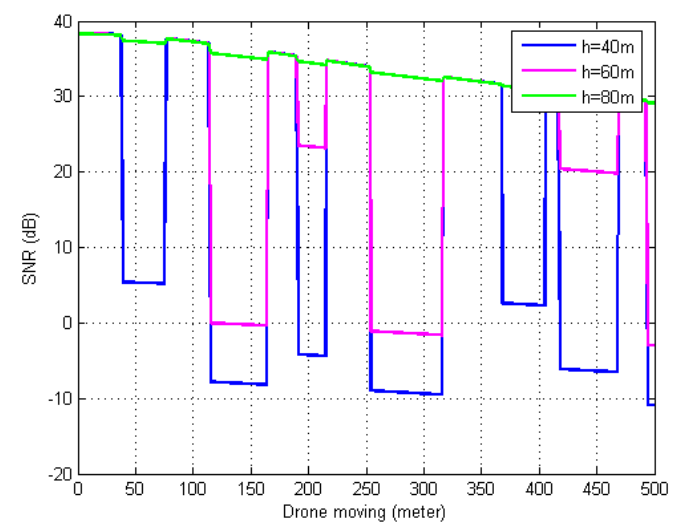

Fig. 9. The communication with transmitter power $30 \mathrm{dBm}$

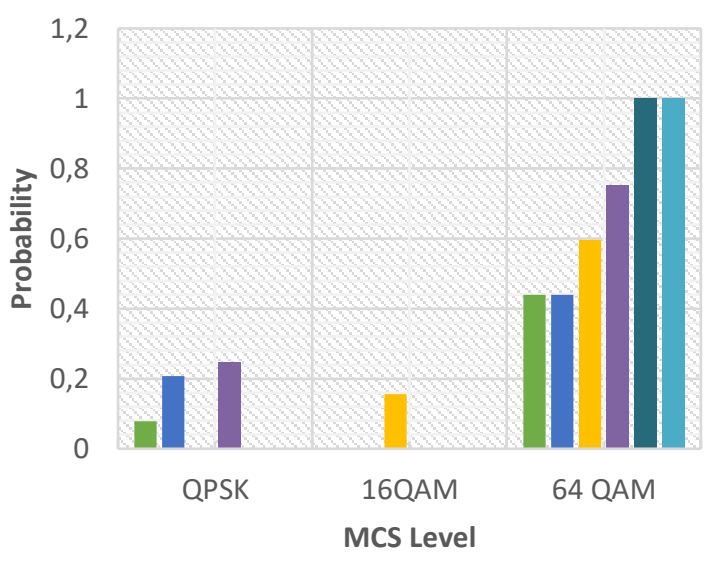

Fig. 10. AMC for drone communication at $10 \mathrm{GHz}$

Figure 8 showed SNR value for LOS and NLOS propagation with a height of flying drone variation for transmitter power $20 \mathrm{dBm}$. The drone used various height, such as 40 meters, 60 meters, and 80 meters. Some data resulted when the height of flying drone 40 meters and transmitter power $20 \mathrm{dBm}$ such as drone location of 20 meters obtained SNR LOS $28.31 \mathrm{dBm}$ that used AMC of 64 QAM code rate 3/4, drone location of 50 meters obtained SNR NLOS $-4,6 \mathrm{dBm}$ that used AMC of QPSK code rate 1/8. Drone location of 400 meters obtained SNR NLOS -7.64 $\mathrm{dBm}$. Some data resulted when the height of flying drone 80 meters and transmitter power $20 \mathrm{dBm}$ such as drone location of 20 meters obtained SNR LOS $28.31 \mathrm{dBm}$ that used AMC of 64 QAM code rate 3/4, drone location of 50 meters obtained SNR LOS 27.29 $\mathrm{dBm}$ that used AMC of 64 QAM code rate 3/4. Drone location of 400 meters obtained SNR LOS $20.84 \mathrm{dBm}$ that used AMC of 64 QAM code rate 3/4.

Figure 9 showed SNR values for LOS and NLOS with a height of flying drone variation at transmitter power $30 \mathrm{dBm}$. The drone used various heights, such as 40 meters, 60 meters, and 80 meters. Some data resulted when the height of flying drone 40 meters and transmitter power $30 \mathrm{dBm}$ such as drone location of 20 meters obtained SNR LOS $38.21 \mathrm{dBm}$ that used AMC of 64 QAM code rate 3/4, drone location of 50 meters obtained SNR NLOS $5.33 \mathrm{dBm}$ that used AMC of QPSK code rate 2/3. Drone location of 400 meters obtained SNR NLOS $2.33 \mathrm{dBm}$ that used AMC of QPSK code rate $1 / 2$. Some data resulted when a height of flying drone 80 meters and transmitter power $30 \mathrm{dBm}$ such as drone location of 20 meters obtained SNR LOS $38.21 \mathrm{dBm}$ that used AMC of 64 QAM code rate 3/4, drone location of 50 meters obtained SNR NLOS $37.29 \mathrm{dBm}$ that used AMC of 64 QAM code rate 3/4. Drone location of 400 meters obtained SNR NLOS $30.84 \mathrm{dBm}$ that used AMC of 64 QAM code rate 3/4. 
Table 1. Detail for Fig.10

\begin{tabular}{|c|c|c|c|}
\hline & \multicolumn{3}{|c|}{ Probability } \\
\hline & QPSK & 16QAM & 64QAM \\
\hline $\begin{array}{c}\mathrm{h}=40 \mathrm{~m} ; \\
\mathrm{pt}=20 \mathrm{dBm}\end{array}$ & 0.078 & 0 & 0.441 \\
\hline $\begin{array}{c}\mathrm{h}=60 \mathrm{~m} ; \\
\mathrm{pt}=20 \mathrm{dBm}\end{array}$ & 0.208 & 0 & 0.441 \\
\hline $\begin{array}{c}\mathrm{h}=80 \mathrm{~m} ; \\
\mathrm{pt}=20 \mathrm{dBm}\end{array}$ & 0 & 0.156 & 0.597 \\
\hline $\begin{array}{c}\mathrm{h}=40 \mathrm{~m} ; \\
\mathrm{pt}=30 \mathrm{dBm}\end{array}$ & 0.247 & 0 & 0.753 \\
\hline $\begin{array}{c}\mathrm{h}=60 \mathrm{~m} ; \\
\mathrm{pt}=30 \mathrm{dBm}\end{array}$ & 0 & 0 & 1 \\
\hline $\begin{array}{c}\mathrm{h}=80 \mathrm{~m} \\
\mathrm{pt}=30 \mathrm{dBm}\end{array}$ & 0 & 0 & 1 \\
\hline
\end{tabular}

Figure 10 showed the MCS probability obtained from communication propagation between drone and RBS. Probability 1 indicates the communication occurred with the best condition. The exact value for Fig. 10 could see in Table 1. This research used MCS such as QPSK, 16 QAM, and 64 QAM. Some data resulted when the height of flying drone 40 meters with transmitter power $20 \mathrm{dBm}$ obtained probability QPSK of 0.078 and 64 QAM of 0.44 . When the flying drone's height 80 meters with a transmitter power of $20 \mathrm{dBm}$ obtained, a probability of 16 QAM of 0.156 and 64 QAM of 0.597. Some data resulted when the height of flying drone 40 meters with a transmitter power $30 \mathrm{dBm}$ obtained probability QPSK of 0.2 and 64 QAM of 0.753 . When the flying drone's height 80 meters with a transmitter power of $30 \mathrm{dBm}$ obtained a probability of 64 QAM of 1 . The increase of height of flying drone could influence SNR value communication, so communication obstacle become decrease. The drone track resulted coverage percentages such as the height of flying drone 40 meters with transmitter power $20 \mathrm{dBm}$ obtained $51.9 \%$, height of flying drone 60 meters with a transmitter power of $20 \mathrm{dBm}$ obtained $64.8 \%$, the height of flying drone 80 meters with transmitter power $20 \mathrm{dBm}$ obtained $75.3 \%$, height of flying drone 40 meters, 60 meters, and 80 meters with transmitter power $30 \mathrm{dBm}$ obtained $100 \%$.

\section{DISCUSSION}

The Research result of communication propagation analyzed communication between drone and base station. Figure 6 analyzed the drone communication propagation with LOS and NLOS conditions when used transmitter power $20 \mathrm{dBm}$. When the drone moving at $20 \mathrm{dBm}$ obtained LOS distances 193.39 meters, some data, and when the drone was moving at 400 meters obtained LOS distances 433.84 meters and NLOS distances 451.52 meters. That NLOS propagation showed more increase of communication distance between drone and RBS. Figure 8 was analyzed about SNR value for
LOS and NLOS propagation when used transmitter power $20 \mathrm{dBm}$. Some data resulted when a drone with the height of flying drone 40 meters such as drone location of 20 meters obtained SNR LOS $28.31 \mathrm{dBm}$ that used AMC of 64 QAM code rate 3/4, and drone location of 400 meters obtained SNR NLOS -7.64 $\mathrm{dBm}$. Some data resulted when the height of flying drone 80 meters, such as drone location of 20 meters obtained SNR LOS $28.31 \mathrm{dBm}$ that used AMC of 64 QAM code rate 3/4, and drone location of 400 meters obtained SNR LOS $20.84 \mathrm{dBm}$ that used AMC of 64 QAM code rate $3 / 4$. Figure 8 and 9 obtained a higher position of the flying drone. The more SNR will increase.

Figure 10 was analyzed about MCS probability from communication propagation. Some data resulted with transmitter $20 \mathrm{dBm}$ when the height of flying drone 40 meters obtained probability QPSK of 0.078 and 64 QAM of 0.44 , and when the height of flying drone 80 meters obtained probability 16QAM of 0.156 and 64 QAM of 0.597 . Some data with transmitter $30 \mathrm{dBm}$ resulted when the height of flying drone 40 meters obtained probability QPSK of 0.2 and 64 QAM of 0.753 . When the height of the flying drone 80 meters obtained probability of 64 QAM of 1 , the increase of height of the flying drone could influence SNR value communication, so communication obstacle become decrease. The track communication of drone analyzed coverage percentages of communication with 40 meters, 60 meters, and 80 meters. The coverage percentages of communication when used height of flying drone 40 meters, 60 meters, and 80 meters with transmitter power $30 \mathrm{dBm}$ obtained $100 \%$. The increase in the height of flying drones could influence the coverage percentage of communication.

\section{CONCLUSION}

This section described the research conclusion about communication propagation between drones and RBS. This research aimed to analyse drone communication propagation around a building environment with the cellular network for remote drone from a long distance. The communication frequency used $10 \mathrm{GHz}$. That frequency was influenced by attenuation atmospheric. The drone used various heights, such as 40 meters, 60 meters, and 80 meters. The transmitter power used $20 \mathrm{dBm}$ and $30 \mathrm{dBm}$. The single knife-edge method is used for the diffraction mechanism. The communication propagation showed SNR value for the height of flying drone variation. Some data that resulted in more drone height obtained more coverage percentage, probability modulation, and SNR value. The necessity with drone communication used video, pictures, sensor data, etc. The height of the flying drone position is adjusted with location and necessity. 


\section{REFERENCES}

[1] A.C. Eska, "Propagation of Mobile Communication with Tree Obstacle used OFDM-QAM at $10 \mathrm{GHz}$, J. INFOTEL, vol.11, no.3, pp.88-92, Sep. 2019.

[2] A.C. Eska, "The Communication System of Building from Outdoor to Indoor with $\mathrm{AMC}$ at 10 GHz," J. INFOTEL, vol.12, no.1, pp. 13-17, Apr. 2020.

[3] G. Castro, R. Feick, M. Rodriguez, R. Vanezuela, and D. Chizhik, "Outdoor-to-Indoor Empirical Path Loss Models : Analysis For Pico and Femto Cells in Street Canyons," IEEE Wireless Communications Letters, vol.6, no.4,pp. 1-1, June. 2017.

[4] G. Bartoli, R. Fantacci, D. Marabissi, and M. Pucci, "Resource Allocation Schemes for Cognitive LTEA Femto-cells using Zero Forcing Beamforming and Users Selection," in 2014 IEEE Global Communication Conference, December 8-12, 2014, Austin. IEEE, 2015.

[5] C. Niu, Y. Li, R. Qingyang, and F. Ye, "Femtocellenhanced multi-target spectrum allocation strategy in LTE-A HetNets," IET communications, vol. 11, no. 6, pp.887-896, Apr. 2016.

[6] A.C. Eska, "Propagasi Komunikasi Radio Base Station Femtocell padaTiang Lampu Jalan Frekuensi $10 \mathrm{GHz}$," J. INFOTEL, vol.9, no.4, pp. 382-386, Nov. 2017.

[7] A.C. Eska, "Pengaruh Code Rate untuk Komunikasi RBS Femtocell Frekuensi $47 \mathrm{GHz}$ pada Tiang Lampu Jalan," J. INFOTEL, vol.9, no.4, pp. 412415, Nov. 2017.

[8] G.R. MacCartney, T.S. Rappaport, S. Sun, and S. Deng, "Indoor Office Wideband Millimeter Wave Propagation Measurements and Channel Models at 28 and $73 \mathrm{GHz}$ for Ultra-Dense 5G Wireless Networks," IEEE Access, vol.3, pp. 2388-2424, Oct. 2015.

[9] A.C. Eska, "Determination of MS Location through Building Using AoA Method of Frequency 47 GHz," IJITEE, vol.1, no.3, pp. 76-80, Sept. 2017.

[10] G.R. MacCartney, and T.S. Rappaport, "MillimeterWave Base Station Diversity for $5 \mathrm{G}$ Coordinated Multipoint (CoMP) Applications," IEEE Transactions on Wireless Communications, vol.18, no.7, pp. 3395-3410, July. 2019.

[11] A. Lukowa, V. Venkatasubramanian, "Dynamic InBand Selft-Backhauling with Interference Cancellation," in IEEE Conference on Standards for Communications and Networking (CSCN), October 29-31, 2018, Paris. IEEE, 2018.

[12] T.S. Rappaport, G.R. MacCartney, S. Sun, H. Yan, and S. Deng, "Small-Scale, Local Area, and Transitional Millimeter Wave Propagation for 5G Communications," IEEE Transactions on Antennas and Propagation, vol. 65, no.12, pp. 6474-6490, Dec. 2017.

[13] T.S. Rappaport, Y. Xing, G.R. MacCartney, A.F Molisch, and E. Mellios, J. Zhang, "Overview of Millimeter Wave Communications for FifthGeneration (5G) Wireless Networks-With a Focus on Propagation Models," IEEE Transactions on Antennas and Propagation, vol. 65, no.12, pp. 6213-6227, Dec. 2017.

[14] T.S. Rappaport, E.B. Dor, J.N. Murdock, and Y. Qiao, "38 GHz and $60 \mathrm{GHz}$ Angle-dependent Propagation for Cellular \& Peer-to-Peer Wireless Communications," in IEEE ICC Wireless Communications Symposium, June 10-15, 2012, Ottawa. IEEE, 2012.

[15] A. Lukowa, V. Venkatasubramanian, P. Marsch,"Dynamic Self - Backhauling in 5G Networks," in IEEE 29th Annual International Symposium on PIMRC, September 9-12, 2018, Bologna. IEEE, 2018

[16] A.C. Eska, "Multipath Effects in Building Environment Toward Bandwidth Enhancement for Mobile Communication of $47 \mathrm{GHz}$ Frequency," J. INFOTEL, vol. 10, no.1, pp.39-44, Feb. 2018.

[17] A.Lukowa, V. Venkatasubramanian, "Dynamic Inband Self-backhauling for 5G Systems with Intercell Resource Coordination," International Journal of Wireless Information Networks, vol. 26, no. 4, pp. 319-330, Dec. 2019.

[18] A.C. Eska, "Adaptive Modulation and Coding around Building Environment for Mobile Station Communication at The Train," EMITTER, vol.6, no.2, pp. 386-394, Dec. 2018.

[19] A.C. Eska, and G. Hendrantoro, "Preliminary study on the effect of building-induced diffraction upon millimeter wave mobile communications systems with macrodiversity," in $20127^{\text {th }}$ TSSA, Oct 30-31, 2012, Denpasar. IEEE, 2012.

[20] A.C. Eska, "Komunikasi Bergerak Frekuensi 2.3 $\mathrm{GHz}$ Melewati Pepohonan Menggunakan Metode Giovanelli Knife Edge," J. INFOTEL, vol. 8, no.1, pp.94-99, May. 2016.

[21] T.W. Wu, and C.D. Chung, "Spectrally Precoded DFT-Based OFDM and OFDMA with Oversampling," IEEE Transactions on Vehicular Technology, vol. 63, no.6, pp. 2769-2783, July. 2014.

[22] A. Shanin, and H. Arslan, "Edge Windowing for OFDM Based Systems," IEEE Communications Letters, vol. 15, no.11, pp. 1208-1211, Nov. 2011.

[23] S. Wang, "Efficient Resource Allocation Algorithm for Cognitive OFDM Systems," IEEE Communications Letters, vol. 14, no.8, pp. 725-727, August. 2010.

[24] J.S. Seybold, Introduction to $R F$ Propagation. Hoboken, New Jersey:John Wiley \& Sons, 2005.

[25] A.C. Eska, Jaringan Komunikasi Selular, Jember : UPT Percetakan \& Penerbitan Universitas Jember, 2018.

[26] ITU-R Radio Communication Sector of ITU (Attenuation by atmospheric gases) ITU-R P.67610. Electronic Publication (Geneva), 2013.

[27] LTE System Specifications and their Impact on RF \& Base Band Circuits. Rohde \&Schwarz, 2013. 